\title{
Digital holographic three-dimensional imaging spectrometry and depth imaging properties
}

\author{
Sirawit Teeranutranont* and Kyu Yoshimori \\ Department of Electrical Engineering and Computer Science, Graduate School of Engineering, \\ Iwate University, Ueda 4-3-5, Morioka, Iwate 020-8551, Japan
}

\begin{abstract}
We have studied a method to obtain both three-dimensional (3-D) spatial information and spectral information of a usual polychromatic object simultaneously by making use of a specifically designed two-wavefront folding interferometer and signal processing, including synthetic aperture technique, spectral decomposition, and 3-D image retrieval. The method uses only interferometric techniques and signal processing applied to the interferogram generated by propagated light from the measured object ${ }^{1-2}$. We call the method the digital holographic 3-D imaging spectrometry.

The method is based on measurement of 5-D interferogram. By applying synthetic aperture technique and spectral decomposition to that 5-D interferogram, one obtains a set of complex holograms of different spectral components. From these holograms, 3-D images of multiple spectral components have been retrieved on the basis of the propagation law applied to the 2-D cross-spectral densities. Decomposed continuous spectrum of each light source is also shown to demonstrate a potential applicability to identify materials of a particular part of object under illumination of white light. This paper reports experimental results in retrieving the spectral components of 3-D images of the spatially incoherent light source distribution. The results for depth imaging properties are also demonstrated.
\end{abstract}

Keywords: Digital holography, Interferometric imaging, Spectroscopy, Fourier transforms, Imaging spectrometry, Low coherence holography, Synthetic aperture technique, Two-wavefront folding interferometer

\section{INTRODUCTION}

Over the past several years, we have reported experimental results in retrieving the 3-D position and spectrum of a monochromatic point source ${ }^{3}$, and a pair of monochromatic point sources of different frequencies, located at different positions ${ }^{4}$. In our recent paper, we have presented experimental results of the method applied to retrieve the spectral components of 3-D images for the spatially incoherent polychromatic object ${ }^{5}$. The measured object in the experiment was the three planar light sources of different shapes. Each planar source has different continuous spectra, located at different 3-D positions. As a result, the 3-D images of the three planar light sources for multiple spectral bands are successfully obtained by our method.

It is well known that, the resolution of the transverse directions for the incoherent holography is considered to be determined with the aperture size as in the coherent holography. The reports of longitudinal resolution along depth direction in the incoherent holography is, however, still rare and has not been sufficiently investigated. The primary purpose of this paper is then to present a longitudinal resolution along the optical axis for the retrieved 3-D spatial information of the measured object. For this purpose, we have investigated the retrieved intensity distributions along depth direction (z-axis) of the 3-D images. The experimental results of 3-D imaging of the retrieved image, especially the depth imaging for the spatially incoherent light source distribution with continuous spectrum are also demonstrated.

\section{PRINCIPLE OF METHOD}

\section{Measurement of 5-D interferogram obtained by two-wavefronts folding interferometer}

We begin by introducing the concept of the measurement system of the digital holographic 3-D imaging spectrometry. A main component of this is called the two-wavefront folding interferometer as illustrated in Figure 1.

*sirawit@q1.cis.iwate-u.ac.jp

ICPS 2013: International Conference on Photonics Solutions, edited by Prathan Buranasiri, Sarun Sumriddetchkajorn, Proc. of SPIE Vol. 8883, 88831B · C 2013 SPIE · CCC code: 0277-786X/13/\$18 · doi: 10.1117/12.2022088 
The measured polychromatic light source distribution is placed on the $x-y$ stage. The light wave, propagating from the light source distribution (S) is divided into two parts by the beam splitter (BS). Each wave front, split by the BS, is reversed up to down or right to left by the right-angle-prism $P$ or P'. Those light waves are superposed on BS again. Then an interference-fringe pattern is created. This interference pattern is recorded by the CCD camera at the observation plane. By moving the piezo translator (PZT) on which the prism $\mathrm{P}$ is placed, an optical path difference $Z$ along the $z$ direction is introduced. By moving the $x-y$ stage and the $z$ stage (PZT) stepwise, the positions of the two divided wave fronts are changed and, then, the interference pattern at each position is recorded. The whole data-set should be regarded as a 5 -D interferogram, namely, $I(x, y, \hat{x}, \hat{y}, Z)$. This may be illustrated as arranging the 2-D elemental interference patterns, in the three-dimensional $(\hat{x}, \hat{y}, Z)$ space as shown in Fig. 2.

\section{Synthetic aperture technique to generate reduced volume (3-D) interferogram}

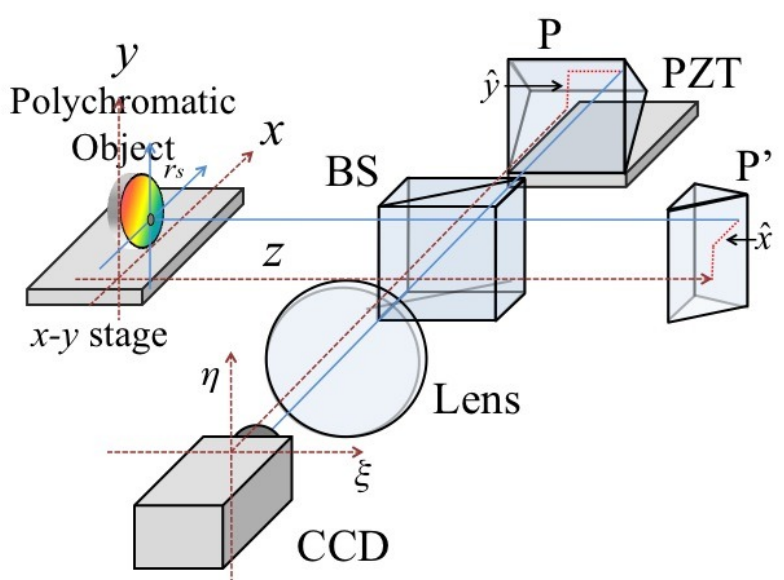

Figure 1. Schematic of the two-wavefront folding interferometer. The optical axes of the interferometer are indicated by solid lines that intersect at the center of the apexes of the right-angle prisms. The Cartesian coordinate system, fixed on the object, is shown by broken lines.

To compute the reduced volume (3-D) interferogram, we apply the synthetic aperture technique to the 5-D interferogram. We will find this interferogram is suitable for retrieving the source information under observation by the selection rule $(\xi=\hat{x}$, and $\eta=-\hat{y})$ and the rearrangement $(X=2 \hat{x}$, and $Y=2 \hat{y})$. The rearranged data set forms the reduced volume (3-D) interferogram (see Fig.3).

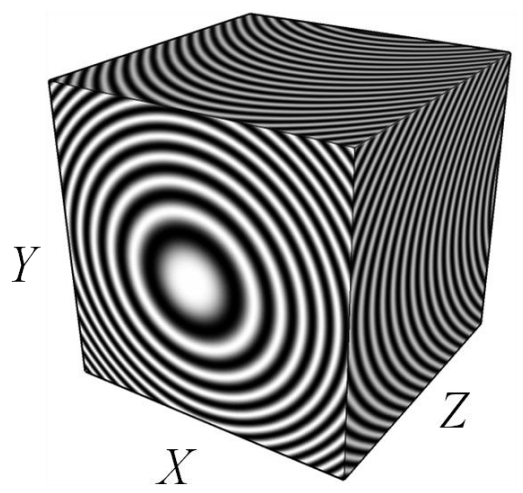

Figure 2. Example of the reduced volume interferogram for monochromatic point source located at the origin of the Cartesian coordinate system.

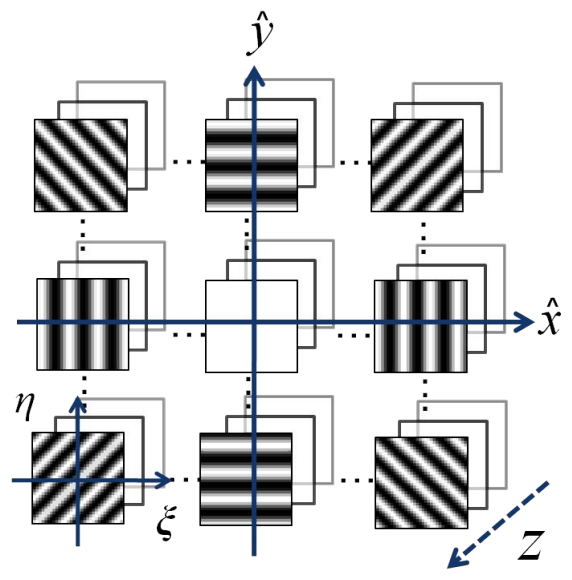

Figure 3. Example of an entire interference data set as the 5-D interferogram: position on each 2-D elemental interference image $(\xi, \eta), x, y$ position of $\mathrm{P}^{\prime}, \mathrm{P}$ apex $(\hat{x}, \hat{y})$, and optical path difference along $z$-direction $(Z)$.

\section{Recovery of a set of complex holograms of different spectral bands}

We find that wavefront information at different wavelengths can be separated from the reduced volume interferogram. Therefore, a set of cross-spectral densities for every spectral component is calculated from the reduced volume interferogram by taking Fourier transform with respect to its depth $(Z)$ as the spectral decomposition technique. 


\section{Retrieval of 3-D image for many spectral bands}

From these incoherent holograms, the 3-D spatial information of the light source distribution at each spectral component is obtained by making use of the basic propagation law applied to the cross spectral density. This cross spectral density is actually equivalent to the incoherent, complex holograms.

\section{EXPERIMENT RESULTS}

In the present experiment, we used LED (Light emitting diode) as the incoherent light source to be measured. The peak wavelength of the continuous spectrum of the light source locates nearly $504 \mathrm{~nm}$. To create planar light source, we attached acrylic stick to the surface of the light source. The cross section on the other side of the acrylic stick is fabricated to a triangular shape. The planar light source is set up on the $x-y$ stage of the two-wavefront folding interferometer. The distance to light source measured from the observation plane is $55 \mathrm{~mm}$. The $x-y$ stage is moved along the $x$ - and $y$ - axes at $12.9 \mu \mathrm{m}$ in every step. The prism P, placed on the piezo translator (PZT), is also moved $0.08 \mu \mathrm{m}$ in every step as well. The total steps of the experiment are $64 \times 64 \times 64$.

Figure 4 shows the continuous spectral profile measured across the observation plane that is acquired from the reduced volume interferogram. The spectral peak around $504 \mathrm{~nm}$ is clearly visible. The phase distribution and absolute value of the crossspectral density at the spectral peak are shown in Figure 5(a) and (b), respectively. This absolute value has its intensity peak at the origin of the observation plane and, then, is proportional to the spectral degree of coherence. We may then call this area the coherence area of the propagating light from the light source to the observation plane. Its extension depends upon the size and the distance of the light source.

Figure 5(c) shows the in-focus image for the light source retrieved from these complex holograms at $55 \mathrm{~mm}$ measured from the observation plane. Within the transverse resolution we find that the size of the image agrees with the actual size of the original light source.

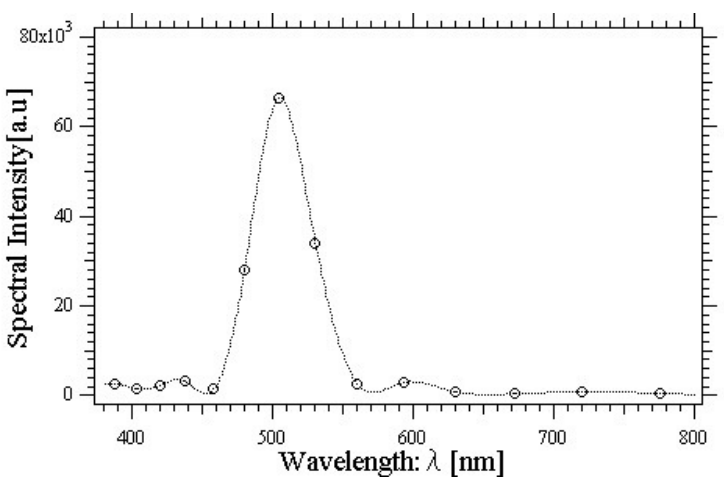

Figure4. Continuous spectral profile recorded on the observation plane. This is obtained by taking Fourier transform of the reduced volume interferogram with respect to $Z$.

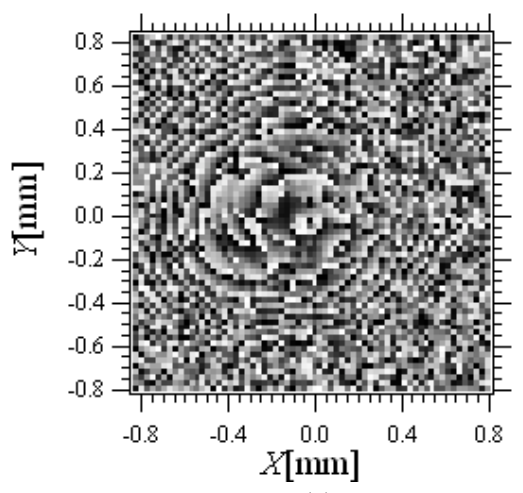

(a)

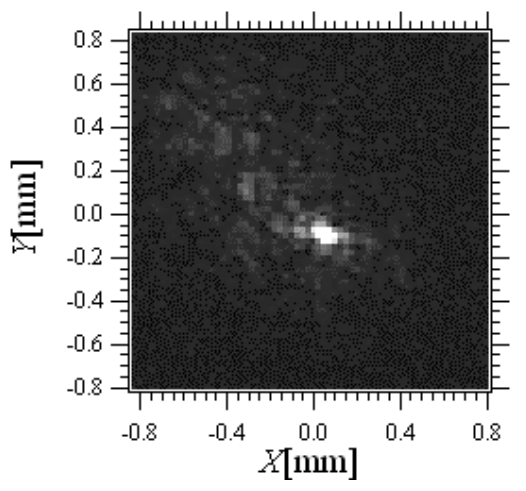

(b)

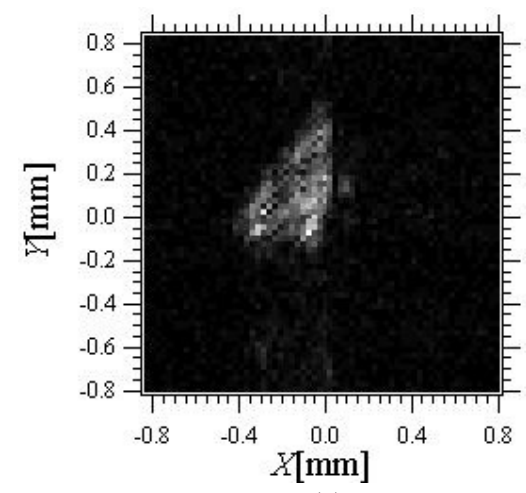

(c)

Figure 5. Phase distribution (a) and absolute value (b) of the cross-spectral density at $\lambda=504 \mathrm{~nm}$. In-focus image (c) over $x-y$ plane at $z=55 \mathrm{~mm}$.

Figure 6 shows the intensity distribution of retrieved image over the $y$-z plane. It is interesting that the dominant light beam backward propagating from the coherence area on the observation plane to the distance of the object forms most parts of the (3-D) source image. In addition, we have plotted the intensity profile along the $z$-axis is shown in Figure 7. The maximum intensity of light source appears close to the actual distance $55 \mathrm{~mm}$ from the observation plane. Thus, the depth information of the light source is properly retrieved. 


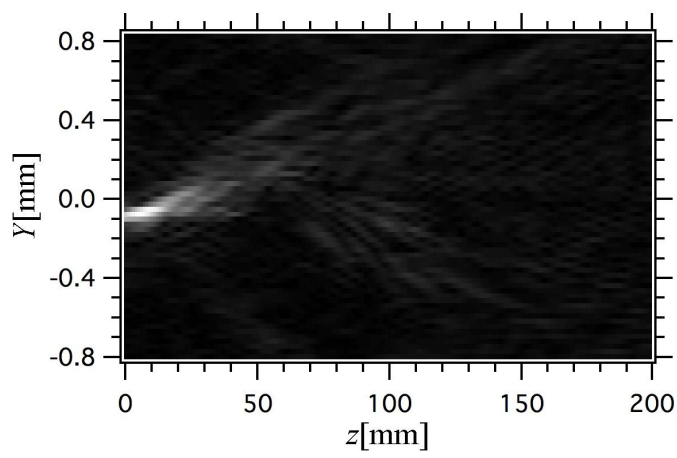

(a) $X=0.1 \mathrm{~mm}$

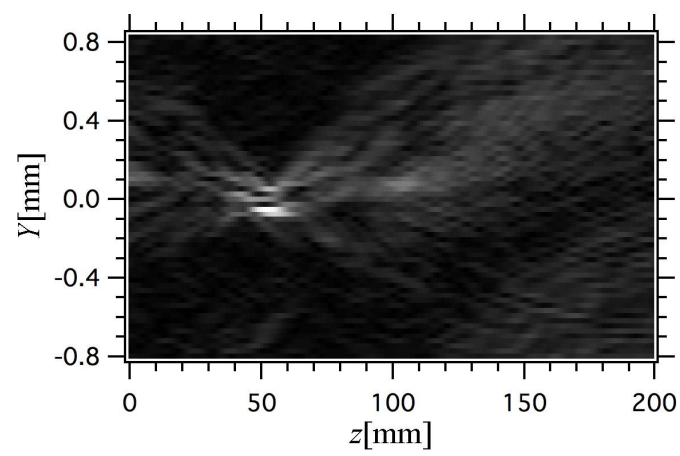

(b) $X=-0.3 \mathrm{~mm}$

Figure 6. Intensity distribution of retrieved image over the $y-z$ plane for the spatially incoherent, planar source.

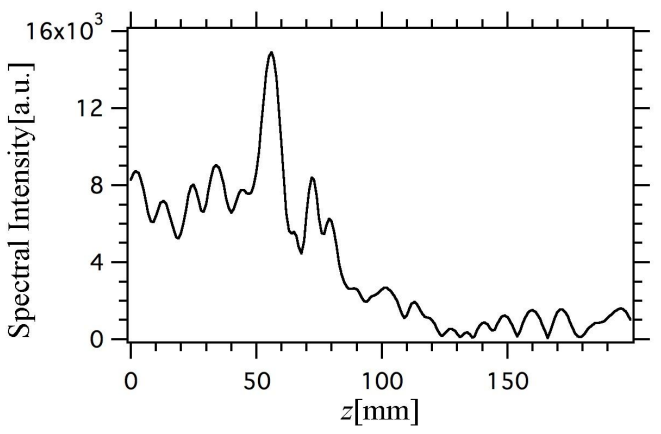

Figure 7. Intensity profile of retrieved image along the $z$-axis at $(X, Y)=(-0.3 \mathrm{~mm},-0.1 \mathrm{~mm})$.

\section{CONCLUSION}

We have shown that, both 3-D spatial information and spectral information of a spatially incoherent, polychromatic light source may be obtained by the digital holographic three-dimensional imaging spectrometry which uses a two-wavefront folding interferometer and signal processing including aperture synthesis, spectral decomposition, and 3-D image retrieval. The retrieved intensity distributions along the depth direction have been investigated.Consequently, we find that the backward propagated light from the coherence area across the observation plane plays a crucial role in generating the dominant parts of 3-D source image.

\section{ACKNOWLEDGMENTS}

This work is supported in part by the Research Foundation for Opto-Science and Technology, and Grands-in-Aid for Scientific Research 25390087 from the Japan Society for the Promotion of Science.

\section{REFERENCES}

[1] K. Yoshimori, "Interferometric spectral imaging for three-dimensional objects illuminated by a natural light source," J. Opt. Soc. Am A 18, 765-770 (2001).

[2] K. Yoshimori, "Passive digital multispectral holography based on synthesis of coherence function," Proc. SPIE 6252, 625221-1-625221-4 (2006).

[3] M. Sasamoto and K. Yoshimori, "First Experimental Report on Fully Passive Interferometric ThreeDimensional Imaging Spectrometry" Jpn. J. Appl. Phys. 48, 09LB03-1-4 (2009).

[4] M. Sasamoto and Kyu Yoshimori, "Three-dimensional imaging spectrometry by fully passive interferometry," Opt. Rev. vol. 19, 29-33 (2012).

[5] S. Teeranutranont and K. Yoshimori, "Digital holographic three-dimensional imaging spectrometry," Appl. Opt. 52, A388-A396 (2013). 